\title{
The Effect of Oxygen Tension in the Medium on the Morphology and Growth Kinetics of Aspergillus nidulans
}

\author{
By B. L. A. CARTER* AND A. T. BULL \\ Department of Microbiology, Queen Elizabeth College, \\ University of London, London W.8
}

(Accepted for publication 24 December 1970)

\begin{abstract}
SUMMARY
In glucose-limited continuous-flow cultures of Aspergillus nidulans, the mean length of hyphal segments and the degree of branching were independent of the dissolved oxygen tension. However, at dissolved oxygen tensions below I $8 \mathrm{mmHg}$ a few large isodiametric cells were observed, and at oxygen tensions below $3.5 \mathrm{mmHg}$ free conidia appeared in the medium (although differentiated conidiophores were not produced).

The critical oxygen tension for Aspergillus nidulans, cultured at $30^{\circ}$ in air-saturated glucose-limited medium (dilution rate $(D)=0.05 \mathrm{~h}^{-1}$ ), was $7 \mathrm{mmHg}$, which suggested that after growth at air saturation the mould could grow without adaptation at oxygen tensions in the medium as low as $7 \mathrm{mmHg}$. However, when the oxygen tension of air-saturated steady-state cultures of the mould was lowered to $30 \mathrm{mmHg}$, or from $30 \mathrm{mmHg}$ to a lesser oxygen tension, a period of adaptation was necessary before the mould culture could again achieve steady-state growth. This adaptation to lower oxygen tensions involved an increased cellular oxidative capacity and a lowered critical oxygen tension for growth. This latter result was indicative of an increased synthesis of the terminal oxidase at low oxygen tensions in the medium.
\end{abstract}

\section{INTRODUCTION}

In batch culture an organism is constantly adapting to an ever-changing environment and therefore it is difficult to obtain precise information on the effect of the environment on the organism. In continuous-flow culture, on the other hand, growth can occur in an unchanging environment and a steady state is established. Therefore continuous-flow cultures have been used in the present investigation to study the effects of dissolved oxygen tension on the morphology and growth kinetics of the filamentous mould Aspergillus nidulans.

That the presence or absence of oxygen affects the metabolic activities of microorganisms is well known; in particular, the presence or absence of mitochondria in yeasts seemingly depends upon the presence or absence of oxygen (Polakis, Bartley \& Meek, 1964). However, only since the introduction of oxygen electrodes and the development of apparatus to control oxygen concentrations in the medium has it been realized that relatively small changes in the oxygen concentration of the medium of aerobic cultures can result in changes in the physiological state of micro-organisms.

* Present address: Laboratory of Molecular Biology, University of Wisconsin, Madison, Wisconsin 53706, U.S.A. 
Wimpenny (1969) has reviewed the present knowledge concerning the influence of oxygen concentration in the medium on yield of organisms, product formation, microbial structure and enzyme activities.

Almost all the critical work on the effect of oxygen on microbial growth has been done with bacteria and yeast; very little work has been done on other eukaryotic organisms. We have previously reported on the effect of various oxygen tensions in the medium on the activities of enzymes of the hexose monophosphate pathway and the Embden-Meyerhof-Parnas pathway in the mould Aspergillus nidulans (Carter \& Bull, 1969). Here we present the results of a study on the effects of different dissolved oxygen tensions (from air saturation to $\mathrm{I} \mathrm{mmHg}$ ) on the morphology and growth kinetics of A. nidulans.

\section{METHODS}

Organism. Strain BWB 224 (Glasgow) of Aspergillus nidulans (Eidam) Wint., a yellow sporing, prototropic recombinant having a velvet morphology.

Culture conditions. The growth medium, continuous-flow culture apparatus and methods of operation and inoculation have been described previously (Carter \& Bull, I969). A constant temperature of $30^{\circ} \pm 0.5^{\circ}$ was maintained throughout all experiments. The methods and apparatus of MacLennan \& Pirt (1966) were used for the automatic control of dissolved oxygen tension of the medium. A Mackereth oxygen electrode model A 15000 (Electronic Instruments Ltd, Richmond, Surrey) was was used as the sensing probe. The electrode was modified for insertion through the fermentor head plate by extending its length with a Perspex sleeve. The electrode was sheathed with a polythene membrane ( 0.0025 in. thick) and produced approximately $2.5 \mu \mathrm{A} / \mathrm{mmHg}$ in water at $30^{\circ}$. The Mackereth electrode was calibrated in sterile medium before inoculation. The medium was allowed to equilibrate with an air flow rate of $200 \mathrm{ml}$./min. until a steady recorder reading was obtained. Oxygen-free nitrogen was used to determine zero dissolved oxygen tension, and the linearity of the electrode was checked with two other gas mixtures of known composition, usually $5 \%$ and $10 \%(\mathrm{w} / \mathrm{v})$ oxygen in nitrogen (British Oxygen Co., London). Oxygen and carbon dioxide concentrations in the effluent gases from the fermentor were determined by methods which we have reported previously (Carter \& Bull, 1969). At the end of each experiment the culture was discarded, the fermentor flushed with sterile medium and the electrode recalibrated to check for drift. In all experiments the drift was negligible.

Oxygen and $\mathrm{pH}$ electrodes were sterilized by ethylene oxide according to the method of Callow \& Pirt (1956).

Oxygen uptake methods. Oxygen uptake rates of freshly harvested mycelia were measured in an oxygen electrode cell by a model 777 oxygen analyser (Beckman Instruments Inc., California, U.S.A.) coupled to a Speedomax type 'W' model S, 0 to $100 \mathrm{mV}$ potentiometric recorder (Leeds and Northrup Ltd, Birmingham) having a response time of I sec. (nominal). The design of the oxygen electrode cell (Fig. I) was based on that of Shugarman \& Appleman (1967). The electrode cell was machined from Perspex and cemented together with chloroform. The reaction chamber was designed to hold $3.5 \mathrm{ml}$. of mycelial suspension or enzyme solution. A water-jacket ensured that the reaction chamber could be maintained at a constant temperature.

Adequate mixing, essential for polarographic measurements, was achieved by using 
a magnetic stirrer. The stirrer bar was made from a piece of wire, $0.5 \mathrm{~mm}$. diam. and about $1 \cdot 0 \mathrm{~cm}$. long, sealed in glass capillary tubing.

A straight hole $(1.0 \mathrm{~mm}$. diam.) was drilled at an angle through the wall of the chamber to permit addition of materials via a hypodermic syringe while the apparatus was in operation. This hole was placed so that it penetrated the inside of the reaction chamber about $2.0 \mathrm{~mm}$. from the bottom, an arrangement that ensured that added materials were mixed thoroughly and quickly. There was no noticeable diffusion through the entry port despite its access to the atmosphere.

A

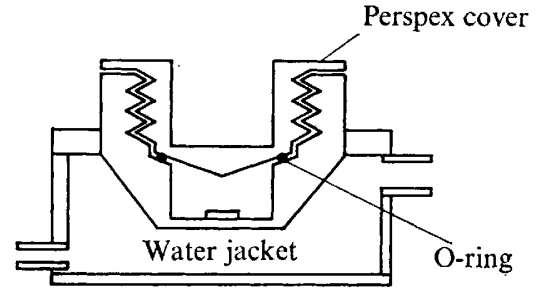

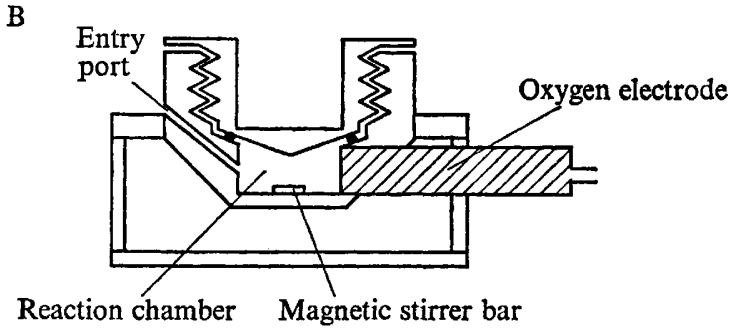

Fig. I. The oxygen electrode cell. The electrode cell was operated at a constant temperature maintained by means of a flow-through water-jacket connected to a thermostatically controlled water bath. The cell is shown in two cross-sectional elevations, B being at right angles to A. Half size.

The oxygen electrode (Beckman Instruments Ltd, Glenrothes, Fife, Scotland) was calibrated daily at $30^{\circ}$. The analyser incorporated a circuit whereby the recorder could be calibrated from zero oxygen to air saturation and be made to fit the full scale of the recorder chart. The zero oxygen reading was determined by adding a few crystals of sodium dithionite to the medium in the oxygen electrode cell at $30^{\circ}$; the oxygen tension of air saturated medium at $30^{\circ}$ was obtained by bubbling air through the medium until saturation was attained. Linearity of electrode response was checked with $5 \%$ and $10 \%$ oxygen in nitrogen (British Oxygen Co.).

\section{Morphology}

RESULTS

In stirred fermentors Aspergillus nidulans produced filamentous growth in which the apical cells were full of cytoplasm and the older cells were vacuolated. This morphology is typical of actively growing mycelia. Batch cultures of the mould made in a small glass fermentor (Quickfit-Quartz, Stone, Staffs.; Carter \& Bull, 1969) produced a mean hyphal segment length (defined as the distance from the apex to the first branch point) of $210 \mu \mathrm{m}$. However, batch cultures made in the chemostat equipment prior to the establishment of continuous-flow conditions produced a much shorter mean hyphal segment length, 50 to $70 \mu \mathrm{m}$. and a more branched mycelium. Probably the closer septation observed under these latter conditions produced enhanced mechanical strength and presumably reflected an adaptation to the increased shearing stresses presented by the chemostat stirrer.

Cultures in which the specific growth rate $(\mu)$ was held constant $\left(\mu=0.05 \mathrm{~h}^{-1}\right)$ and the dissolved oxygen tension varied from I to $156 \mathrm{mmHg}$ developed a filamentous growth morphology. Both the mean hyphal segment length and the degree of branching appeared to be independent of the dissolved oxygen tension. 
Yeastlike differentiation was not observed even at the lowest oxygen tensions investigated (I mmHg). However, at oxygen tensions below $18 \mathrm{mmHg}$ a few large, approximately isodiametric cells were observed. At even lower oxygen tensions ( $3.5 \mathrm{mmHg}$ and less) free conidia appeared in the medium but differentiated conidiophores were not produced.

The fine structure of Aspergillus nidulans, as revealed in ultra-thin sections, was typical of ascomycetous fungi that had been grown in environments in which the dissolved oxygen tension was above the critical oxygen tension (see below). The cells were multinucleate and contained many mitochondria; the cytoplasm was dense but the endoplasmic reticulum was quite sparse. Lomasomes also were recognizable between the protoplasmic membrane and the multilayered cell wall. Vacuoles were visible in subapical cells and occasionally more than one vacuole per cell was present. The oxidative capacity of $A$. nidulans grown at oxygen tensions of $2 \mathrm{mmHg}$ was greater than when grown in an air-saturated medium (see below), but this phenomenon was not accompanied by a significant increase in the number of cristae per mitochondrion or in the apparent number of mitochondria per cell. Electron micrographs of thinsectioned hyphae grown at the critical oxygen tension of $\mathrm{I} \cdot 75 \mathrm{mmHg}$ revealed extensive nuclear and mitochondrial degradation.

\section{Growth kinetics}

The growth kinetics of Aspergillus nidulans were studied at a variety of steady-state oxygen tensions. The specific growth rate of the fungus was controlled at $0.05 \mathrm{~h}^{-1}$, in a glucose-limited chemostat culture, and the oxygen tension of the medium was varied using the oxygen-control system described above. Sufficient time was allowed for possible adaptation of the fungus to each oxygen tension before measurements were made. In practice, the establishment of steady-state conditions was taken to indicate complete adaptation.

The effect of oxygen tension in the medium on the specific rate of oxygen uptake is shown in Fig. 2. These data suggest that the oxygen tension in the medium had little effect on the growth kinetics of Aspergillus nidulans in the range $156 \mathrm{mmHg}$ (= air saturation) to $10 \mathrm{mmHg}$, and probably lower. The oxygen uptake rate was reasonably constant down to $1.75 \mathrm{mmHg}$ but then fell markedly at oxygen tensions below $\mathrm{I} \cdot 75 \mathrm{mmHg}$. This value of $\mathrm{I} \cdot 75 \mathrm{mmHg}$ is the critical oxygen tension. Above this value oxygen uptake rates were independent of the oxygen tension of the medium. Because the observations in Fig. 2 were made from steady-state cultures, we cannot decide from the data whether the fungus required a period of adaptation before it exhibited the same growth kinetics at hypobaric oxygen tensions as it did at air saturation. To investigate this problem further, the fungus grown in an air-saturated medium ( $D=0.05 \mathrm{~h}^{-1}$ ) was removed from the chemostat, collected by centrifugation, resuspended in fresh, air-saturated medium, incubated (without aeration) at $30^{\circ}$ in the oxygen electrode cell and the oxygen uptake rates measured using the exhaustion-curve technique. Clearly (Fig. 3) the oxygen uptake rate of the fungus grown in the airsaturated medium, in the chemostat, was independent of dissolved oxygen tension at values well below a medium oxygen tension of $30 \mathrm{mmHg}$. These results suggested that $A$. nidulans could grow at an oxygen tension of $30 \mathrm{mmHg}$, after previous growth at an oxygen tension of $156 \mathrm{mmHg}$, without a period of adaptation. However, had no adaptation been required to accommodate a change in the oxygen tension of the 
medium from $156 \mathrm{mmHg}$ to $30 \mathrm{mmHg}$, then the oxygen uptake rate in the chemostat should have remained constant and the steady state undisturbed. In practice the oxygen uptake rate of the culture fell when the medium oxygen tension was reduced, although after a transient state (during which the fungus adapted to the lower oxygen tension) a new steady state was established in which the oxygen-uptake rate of the culture was the same as that observed at the higher medium oxygen tension (Table I).

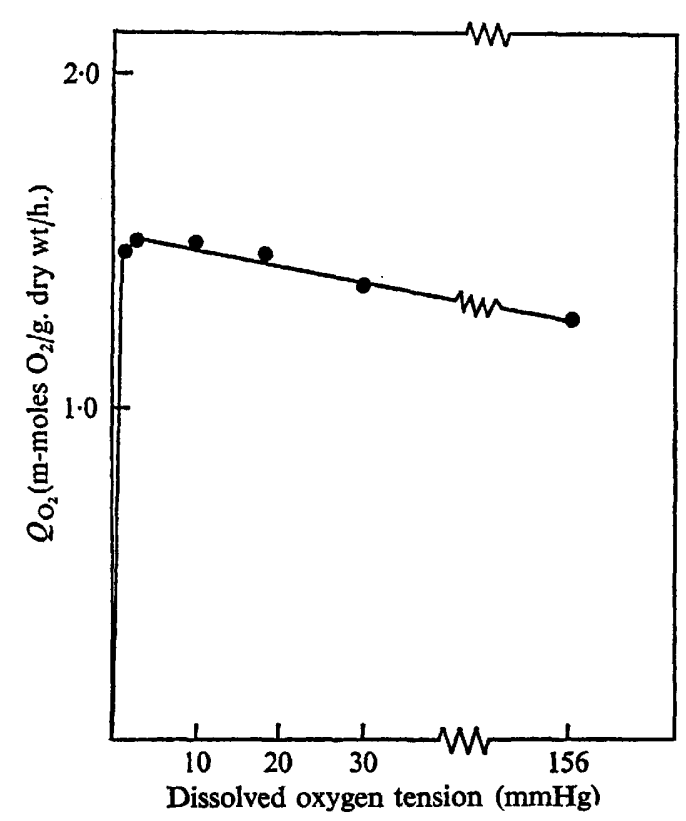

Fig. 2

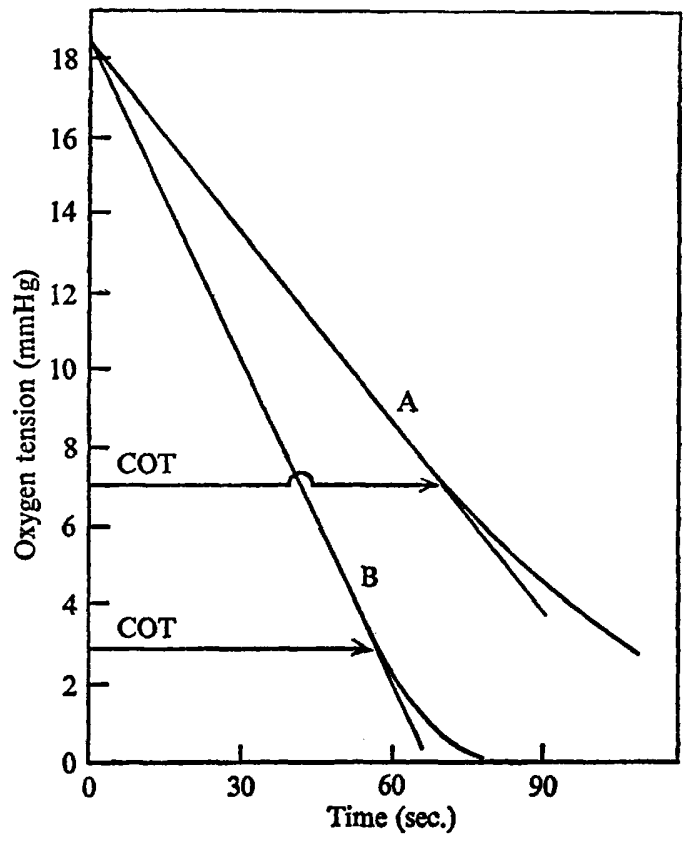

Fig. 3

Fig. 2. The effect of dissolved oxygen tension on the specific rate of oxygen uptake. At dissolved oxygen tensions below $1.75 \mathrm{mmHg}$ a steady state was not obtained and the oxygen uptake rate fell. Below $1.75 \mathrm{mmHg}$ the oxygen uptake rate was no longer independent of oxygen tension in the medium and therefore this value of $1 \cdot 75 \mathrm{mmHg}$ represents the critical oxygen tension. The specific rate of oxygen uptake was calculated from measurements of the oxygen content of the effluent gas from the chemostat.

Fig. 3. Exhaustion curves. Mycelial samples were removed from the chemostat, collected by centrifugation, resuspended in fresh, air-saturated medium incubated (without aeration) at $30^{\circ}$ in the oxygen electrode cell and the oxygen uptake rates calculated from measurements of the disappearance of oxygen in the medium. (A) Mycelia previously grown at an oxygen tension of $156 \mathrm{mmHg}$ (air saturation). (B) Mycelia previously grown at an oxygen tension of $2 \mathrm{mmHg}$. At oxygen tensions below the critical oxygen tension (COT) oxygen uptake rate was no longer independent of the oxygen tensions in the medium.

Table 2 shows further evidence of the adaptation of Aspergillus nidulans to lower oxygen tensions. In steady-state cultures of $A$. nidulans in air-saturated medium $\left(D=0.05 \mathrm{~h}^{-1}\right)$ the residual glucose concentration of the medium usually was less than $0.12 \mathrm{mg} . / \mathrm{ml}$. However, when the medium oxygen tension was reduced to $30 \mathrm{mmHg}$, the steady state was disturbed and the glucose concentration of the medium increased to over $2 \mathrm{mg}$. $/ \mathrm{ml}$. This indicated that the fungus was unable to oxidize all the available glucose. As the fungus adapted to the new oxygen tension it was able 
to oxidize more of the glucose and the steady-state glucose concentration fell to $0.48 \mathrm{mg}$. $/ \mathrm{ml}$. These data suggest that adaptation involving an increase in the oxidative capacity of the fungus may occur when the fungus is transferred from a medium of high oxygen tension ( $156 \mathrm{mmHg}$ ) to a medium of low oxygen tension $(30 \mathrm{mmHg}$ ). To investigate this hypothesis further, measurements were made of the oxygen uptake rate of steady state $A$. nidulans samples. The data presented in Table 3 show that the oxidative capacity of the fungus (oxygen uptake rate with all medium constituents in excess) increased as the oxygen tension in the medium was decreased. Thus one outcome of adaptation to a decreased medium oxygen tension was an increased oxidative capacity.

Table I. The effect of dissolved oxygen tension (DOT) on the specific utilization rate

$$
\left(Q_{\mathrm{O}_{2}}\right) \text { of Aspergillus nidulans }
$$

A culture doubling of $\mathrm{I} \cdot \mathrm{O}$ indicates that, after the steady state has been perturbed, the mould growing at a specific growth rate of $0.05 \mathrm{~h}^{-1}$ had doubled in mass. $Q_{\mathrm{O}_{2}}$ was calculated from measurements of the oxygen content of effluent gas from the chemostat. Transition-state measurements were taken at various periods after the steady state was perturbed by lowering the medium oxygen tension. In practice, full adaptation occurred after three culture doublings, and a steady state was then re-established.

$\begin{array}{ccc}\text { DOT (mmHg) } & Q_{\mathrm{O}_{2}} & \begin{array}{c}\text { No. of culture } \\ \text { doublings }\end{array} \\ \text { I56 (steady state) } & \mathrm{I} \cdot 22 & - \\ \text { 30 (transition state) } & 0 \cdot 89 & \mathrm{I} \cdot 2 \\ \text { 30 (transition state) } & \mathrm{I} \cdot 10 & 2 \cdot 3 \\ \text { 30 (steady state) } & \mathrm{I} \cdot 29 & 3 \cdot 3\end{array}$

Table 2. Residual glucose concentrations in the medium in steady and transition state cultures following a reduction of the dissolved oxygen tension (DOT)

Glucose was determined with a glucose oxidase reagent (Boehringer Mannheim $\mathrm{GmbH}$, Mannheim, Germany). Dilution rate $(D)=0.05 \mathrm{~h}^{-1}$. See Table I for further details.

$\begin{array}{ccc}\text { DOT (mmHg) } & \begin{array}{c}\text { Residual glucose } \\ (\mathrm{mg} \% \%)\end{array} & \begin{array}{c}\text { No. of culture } \\ \text { doublings }\end{array} \\ \text { 156 (steady state) } & 6 \cdot 0 & - \\ \text { 30 (transition state) } & 214 \cdot 0 & 2 \cdot 4 \\ \text { 30 (steady state) } & 48 \cdot 0 & 3 \cdot 6\end{array}$

Table 3. The effect of dissolved oxygen tension (DOT) on the oxidative capacity of Aspergillus nidulans

Samples were removed from the chemostat, centrifuged, incubated with fresh medium in the oxygen electrode cell and the oxygen utilization rate measured with an oxygen electrode. Dilution rate $(D)=0.05 \mathrm{~h}^{-1}$.

$\begin{array}{cc}\text { DOT }(\mathrm{mmHg}) & \begin{array}{c}\text { Oxidative capacity } \\ \left.\text { (mmoles } \mathrm{O}_{2} / \mathrm{g} . / \mathrm{h} .\right)\end{array} \\ \text { I } 56 \text { (steady state) } & \mathrm{I} \cdot 3 \mathrm{I} \\ 2 \text { (steady state) } & 2 \cdot 40\end{array}$

Several workers have calculated values for the critical oxygen tension of a variety of micro-organisms, but only White (1963) has considered the possibility that the critical oxygen tension of an organism may be dependent on environment. In the present investigation 'exhaustion curve' techniques were used to examine the influence 
of the oxygen tension in the medium on the critical oxygen tension of Aspergillus nidulans (Fig. 3). Whenever the oxygen tension was above the critical oxygen tension the oxygen uptake rate was constant, but whenever it was below this critical value oxygen uptake rates were dependent on the oxygen tension in the medium. Using the above technique it was found that the critical oxygen tension of $A$. nidulans was dependent on the oxygen tension of the medium in which the fungus had been grown previously. The critical oxygen tension of $A$. nidulans grown at oxygen tensions of I $56 \mathrm{mmHg}$ was $7 \mathrm{mmHg}$ whereas with cultures grown at oxygen tensions of $2 \mathrm{mmHg}$ the critical value was $2.8 \mathrm{mmHg}$.

\section{DISCUSSION}

Morphological differentiation of Aspergillus nidulans in chemostat cultures was very slight compared with that found in batch cultures. Rippon (1968) observed that filamentous moulds such as Penicillium lilacinum developed budding yeast-like cells when grown in submerged culture under conditions of low redox potential. Aspergillus nidulans did not respond in this manner even at dissolved oxygen tensions as low as $\mathrm{I} \mathrm{mmHg}$. Abnormal morphologies similar to the large, thick-walled cells which we observed at oxygen tensions below $18 \mathrm{mmHg}$ also have been reported in Fusarium oxysporum grown at low oxygen levels (Hollis, 1948) and in Penicillium chrysogenum when grown under highly acid conditions (Bent \& Morton, 1963). Differentiation of these aberrant cells suggests that the fungus is under some physiological stress. Righelato, Trinci, Pirt \& Peat (1968) discovered that asexual spore differentiation occurred in $P$. chrysogenum when the glucose feed rate in chemostat cultures was lowered to, or just above, the maintenance ration. These authors speculated that sporulation was preceded by a qualitative change in glucose catabolism. Our own investigations of conidiation in batch cultures of $A$. nidulans support this view and revealed a maximum operation of the hexose monophosphate pathway immediately prior to conidia production (Carter \& Bull, 1969). Preliminary examination of $A$. nidulans under maintained steady-state conditions failed to reveal the complete sporulative differentiation stages seen in batch cultures and, simultaneously, the hexose monophosphate pathway activity fell to an extremely low level (A. T. Bull, unpublished results).

Results from 'exhaustion curve' studies (Fig. 3) showed that the critical oxygen tensions of cultures previously grown at oxygen tensions of $156 \mathrm{mmHg}$ was $7 \mathrm{mmHg}$ whereas this value was lowered to $2.8 \mathrm{mmHg}$ when the cultures were grown at oxygen tensions of $2 \mathrm{mmHg}$. Neither of these values was as low as the critical oxygen tension observed in the chemostat ( $1 \cdot 75 \mathrm{mmHg}$; see Fig. 2). The values calculated from exhaustion curves and values observed in the chemostat are not necessarily inconsistent. The metabolic rate of Aspergillus nidulans is limited by its oxidative capacity in the 'exhaustion curve' technique because glucose is supplied in excess. In the chemostat, on the other hand, the metabolic rate is limited by the glucose supply rate and this rate may be below the oxidative capacity of the fungus. Fig. 2 indicates that when the critical oxygen tension (as calculated from exhaustion curves) is $2.8 \mathrm{mmHg}$ the fungus can maintain an oxygen uptake rate corresponding to half the oxidative capacity at oxygen tensions below $2.8 \mathrm{mmHg}$. If the critical oxygen tension of the fungus was measured when the metabolic rate was restricted to less than the maximum rate, then a value below $2.8 \mathrm{mmHg}$ for the critical oxygen tension would be recorded. Such a condition may be realized in the chemostat where the metabolic rate of the fungus is 
limited by the glucose supply rate. It is obvious from this discussion that any significant data on critical oxygen tension must include precise information on the growth conditions and must define the experimental conditions used to obtain the data.

The value of $1.75 \mathrm{mmHg}$ for the critical oxygen tension of Aspergillus nidulans is the lowest recorded for a filamentous mould and is the only recorded value which has been calculated from oxygen uptake rates of steady-state cultures. Phillips \& Johnson (I96I) calculated the critical oxygen tension from 'exhaustion curves' of two filamentous moulds grown in aerated batch cultures. The critical oxygen tension was I $2 \mathrm{mmHg}$ for Penicillium chrysogenum and I $\mathrm{mmHg}$ for $A$. niger. In the present study a value of $7 \mathrm{mmHg}$ for the critical oxygen tension was obtained for air-saturated steady-state cultures of $A$. nidulans at $30^{\circ}$ in a glucose-limited chemostat $\left(D=0.05 \mathrm{~h}^{-1}\right)$.

Thus the critical oxygen tension was lower in Aspergillus nidulans previously grown in medium with an oxygen tension of $2 \mathrm{mmHg}$ than when the organisms were grown in air-saturated medium. White (1963) came to a similar conclusion from his studies of Haemophilus parainfluenzae. He found that cell suspensions from poorly aerated cultures had much lower critical oxygen tensions ( $3 \mathrm{mmHg}$ ) than those from vigorously aerated cultures $(76 \mathrm{mmHg})$. The lower critical oxygen tension observed at low oxygen tensions in the medium indicates an enhanced affinity of $A$. nidulans for oxygen which, in turn, may reflect increased synthesis of the terminal oxidase. However, mycelia which had adapted to these low medium oxygen tensions had greater oxidative capacities than mycelia grown at higher medium oxygen tensions. These results suggest that other enzymes concerned with oxidative metabolism are synthesized during the adaptation period and that adaptation does not involve merely an increased synthesis of the terminal oxygen acceptor. The biochemical bases for these observations will be the subject of a later publication.

We wish to thank Mr B. I. Rowley for help with the chemostat apparatus, Mr K. W. Reynolds for making the electrode cell, and Mr D. J. Border and Dr A. P. J. Trinci for assisting our electron-microscope study. An equipment grant from the Central Research Fund of the University of London and Science Research Council support for B.L.A.C. are acknowledged with gratitude.

\section{REFERENCES}

BeNT, W. J. \& Morton, A. G. (1963). Formation and nature of swollen hyphae in Penicillium and related species. Transactions of the British Mycological Society 46, 401 .

Callow, D. S. \& PIRT, S. J. (1956). Automatic control of pH value in cultures of micro-organisms. Journal of General Microbiology r4, 66I-669.

CARTER, B. L. A. \& BuLl, A. T. (1969). Studies of fungal growth and intermediary carbon metabolism in steady state and non-steady state conditions. Biotechnology and Bioengineering 11, 785-804.

Hollis, J. P. (1948). Oxygen and carbon dioxide relations of Fusarium oxysporum Schlect and Fusarium eumartii Carp. Phytopathology 38, 76I-774.

MacLennan, D. J. \& PIRT, S. J. (1966). Automatic control of dissolved oxygen tension in stirred microbial cultures. Journal of General Microbiology 45, 289-302.

Phillips, D. H. \& Johnson, M. J. (196r). Measurement of dissolved oxygen in fermentations. Biotechnology and Bioengineering 3, 26I-270.

Polakis, E. S., Bartley, W. \& MeEk, G. A. (1964). Changes in the structure and enzyme activity of Saccharomyces cerevisiae in response to changes in the environment. Biochemical Journal 90, 369-374. 
Righelato, R. C., Trinci, A. P. J., Pirt, S. J. \& Peat, A. (I968). The influence of maintenance energy and growth rate on the metabolic activity, morphology and conidiation of Penicillium chrysogenum. Journal of General Microbiology 50, 399-412.

RiPPON, J. W. (1968). Monitored environment system to control cell growth, morphology and metabolic rate in fungi by oxidation reduction potentials. Applied Microbiology 16, I 14-I 21 .

Shugarman, D. M. \& Appleman, D. (1967). A simple oxygen electrode chamber and method for measurement of photosynthetic oxygen evolution. Analytical Biochemistry 18, 193-202.

WHITE, D. C. (I963). Factors affecting the affinity for oxygen of cytochrome oxidases in Haemophilus parainfluenzae. Journal of Biological Chemistry 238, 3757-376r.

WIMPENNY, J. W. T. (1969). Oxygen and carbon dioxide as regulators of microbial growth and metabolism. Symposia of the Society for General Microbiology 19, 161-172. 